\title{
On Combinatorial Proofs for Modal Logic
}

\author{
Matteo Acclavio ${ }^{1}$ and Lutz Straßburger ${ }^{2}$ \\ 1 Università Roma Tre http://matteoacclavio.com/Math.html \\ 2 Inria Saclay http://www.lix.polytechnique.fr/Labo/Lutz.Strassburger/
}

\begin{abstract}
In this paper we extend Hughes' combinatorial proofs to modal logics. The crucial ingredient for modeling the modalities is the use of a self-dual non-commutative operator that has first been observed by Retoré through pomset logic. Consequently, we had to generalize the notion of skew fibration from cographs to Guglielmi's relation webs. Our main result is a sound and complete system of combinatorial proofs for all normal and non-normal modal logics in the S4-tesseract. The proof of soundness and completeness is based on the sequent calculus with some added features from deep inference.
\end{abstract}

Keywords: Combinatorial Proofs · Modal Logic · S4-tesseract · Relation Webs · Skew Fibration.

\section{Introduction}

During the last three decades, the proof theory of modal logics has seen enormous progress. We have now access to a systematic treatment of modal logics in display calculus [32], calculus of structures [26]14, labeled systems [25122], hyper sequents [3]18, and nested sequents [5]28|20]. There are focused proof systems for classical and intuitionistic modal logics 788 , and we understand the relation between display calculus and nested sequents [10] and hyper sequents [11].

The motivation for this paper is to take the natural next step in this advancement. After having developed various proof systems, using different formalisms, we are now asking the question: When are two proofs the same?

We are not claiming to provide a final answer to this question, but we propose an approach based on combinatorial proofs, introduced by Hughes [15]16] to address the question of proof identity for classical propositional logic and Hilbert's 24th problem 3130. Via combinatorial proofs, it is finally possible to ask the question of proof identity also for proofs in different proof formalisms; recent research has investigated this for syntactic proofs in sequent calculus [1615, calculus of structures [29, resolution calculus, and analytic tableaux [1].

In classical propositional logic, a combinatorial proof is a skew fibration $f: \mathcal{G} \rightarrow \mathcal{F}$ from an $R B$-cograph $\mathcal{G}$, that can be seen as the "linear part" of the proof, to a cograph $\mathcal{F}$ that encodes the conclusion of the proof. The mapping $f$ precisely captures the information about what is duplicated and deleted in the proof. In terms of syntactic proof systems, this corresponds to the rules of contraction and weakening. 
As an example we show below the combinatorial proof of Pierce's law. On the left we show the conclusion as formula, and on the right as cograph.
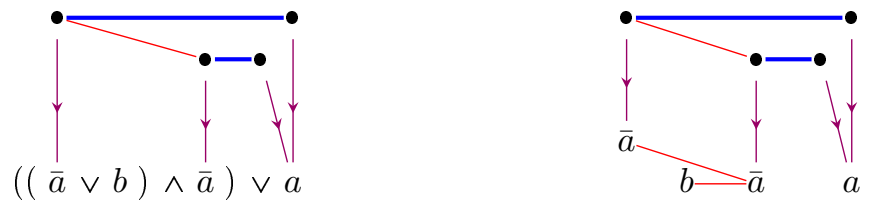

There, the regular (red) R-edges are the edges of the RB-cograph $\mathcal{G}$, and the bold (blue) B-edges represent the linking, corresponding to the instances of the axiom-rule in the sequent calculus. The vertical arrows (purple) represent the mapping $f$.

There is a close correspondence between cographs and formulas composed from atoms via two binary (commutative and associative) connectives, $\wedge$ (and) and $\vee($ or $)$ : the vertices of the cograph are the atom occurrences in the formula, and there is an (undirected) edge between two atom occurrences if their first common ancestor in the formula tree is an $\wedge$, and there is no edge if it is an $\vee$.

For this reason, the cograph-approach works very well for classical propositional logic (CPL) [15/16/27/29] and for multiplicative linear logic (MLL) 24], but it is not obvious how to extend this notion to modalities, which can be seen as unary connectives.

We solve this problem by adding a third non-commutative (self-dual) operation $\triangleleft(s e q)$, that has first been proposed by Retoré in pomset logic 23] and later been studied in the logic BV [12]13. In the corresponding graph, we put a directed edge between two atoms if their first common ancestor in the formula tree is an $\triangleleft$. With this insight we can now represent a formula $\square A$ (resp. $\diamond A$ ) as graph by taking the graph of $A$, add a vertex labeled with $\square$ (resp. $\diamond)$ and add a directed edge from that vertex to every vertex in $A$. This is illustrated in the example below, which is a proof in the modal logic $\mathrm{K}$, and which is a variation of the example in (1) above. As before, on the left the conclusion is written as formula, and on the right as graph.
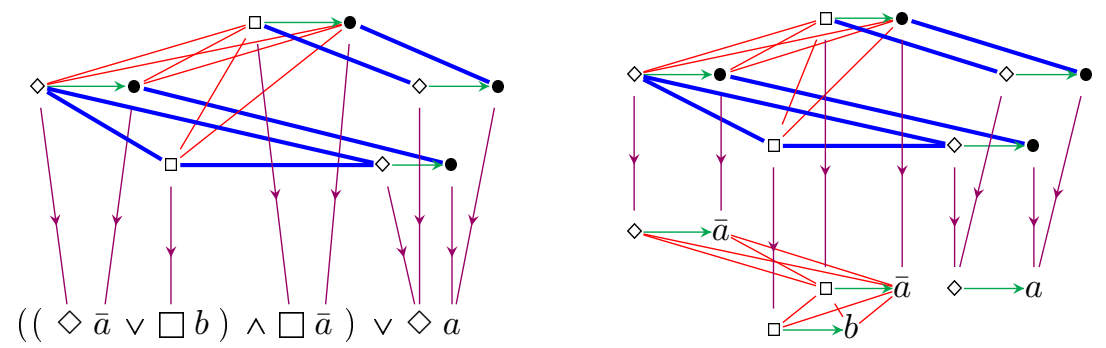

The upstairs graph is now no longer an RB-cograph but an $R G B$-cograph which additionally has directed (green) G-edges. The downstairs graph is a relation web which is a generalization of a cograph to more than two connectives.

The contributions of this paper can now be summarized as follows: we present a notion of combinatorial proof for the modal logics in the S4-plane (shown on the 

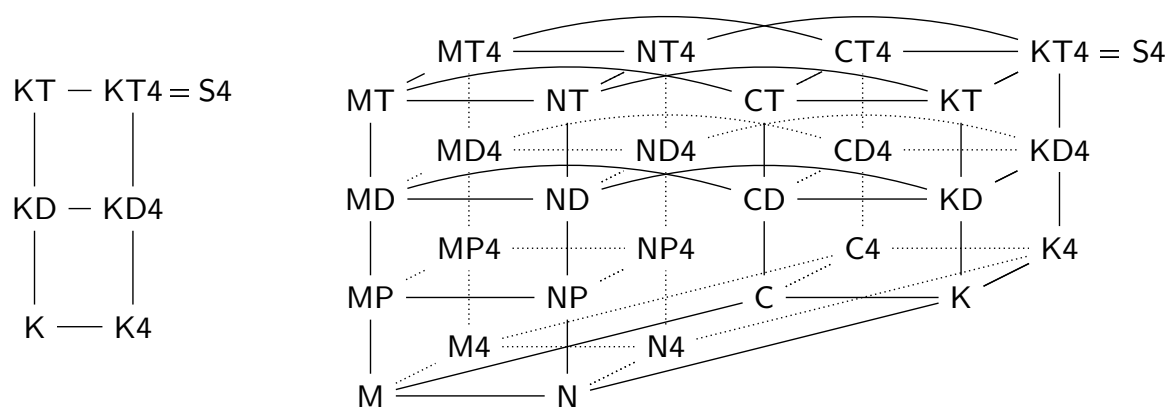

Fig. 1. On the left: the S4-plane and on the right the S4-tesseract

left in Figure 19, and we show how sequent proofs are translated to combinatorial proofs, and that this translation is polynomial in the size of the proof. We then show that these results can be extended to the non-normal modal logics of the S4-tesseract [19] (shown on the right in Figure 1).

We begin by recalling in Section 2 the sequent calculus systems for the modal logics in the S4-plane. Then, in Section 3 we recall the notion of Guglielmi's relation webs [12. Section 5 introduces skew fibrations on relation webs and shows that they correspond to contraction-weakening maps. In Section 4 we introduce the notion of RGB-cograph and show the relation to "linear" proofs in modal logics. The results of Sections 5 and 4 are combined in Section 6 to define combinatorial proofs for the modal logics $\mathrm{K}$ and $\mathrm{KD}$ and show their soundness and completeness. We also show that they form a proof system in the sense of Cook and Reckhow 9. Then, Section 7 shows how to treat modal logics that include the axioms T and/or 4, and finally, in Section 8, we show how our results can be extended to all logics in the S4-tesseract.

\section{Sequent calculus}

We consider the class $\mathcal{K}$ of modal formulas (denoted by $A, B, \ldots$ ) in negation normal form, generated by a countable set $\mathcal{A}=\{a, b, \ldots\}$ of propositional variables and their duals $\overline{\mathcal{A}}=\{\bar{a}, \bar{b}, \ldots\}$ by the following grammar:

$$
A, B::=a|\bar{a}| A \vee B|A \wedge B| \square A|\diamond A| \square \perp \mid \diamond \perp
$$

where $\perp$ stands for the empty formula ${ }^{3}$ An atom is an element of $\mathcal{A} \cup \overline{\mathcal{A}} \cup$ $\{\square \perp, \diamond \perp\}$. A sequent $\Gamma=A_{1}, \ldots, A_{n}$ is a non-empty multiset of formulas, written as list separated by comma.

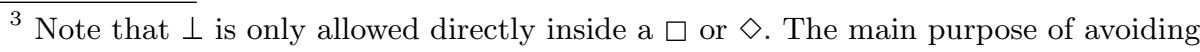
$\perp$ as proper formula is to avoid the empty relation web (to be introduced in the next section). However, we do need formulas $\square \perp$ and $\diamond \perp$ in order to allow weakenings inside $a \square$ or $\diamond$, which is needed to prove the decomposition theorem (stated in Theorem 2.2 below) which in turn is the basis for combinatorial proofs.
} 


$$
\begin{array}{ccccc}
\frac{\Gamma, \bar{a}}{a} \mathrm{ax} & \frac{\Gamma, A, B}{\Gamma, A \vee B} \vee & \frac{\Gamma, A \quad B, \Delta}{\Gamma, A \wedge B, \Delta} \wedge & \frac{\Gamma}{\Gamma, A} \mathrm{w} & \frac{\Gamma, A, A}{\Gamma, A} \mathrm{c} \\
\frac{A, \Gamma}{\square A, \diamond \Gamma} \mathrm{k} & \frac{A, \Gamma}{\diamond A, \diamond \Gamma} \mathrm{d} & \frac{\Gamma, A}{\Gamma, \diamond A} \mathrm{t} & \frac{A, \Gamma, \diamond \Delta}{\square A, \diamond \Gamma, \diamond \Delta} 4_{\mathrm{k}} & \frac{A, \Gamma, \diamond \Delta}{\diamond A, \diamond \Gamma, \diamond \Delta} 4_{\mathrm{dk}}
\end{array}
$$

Fig. 2. Sequent calculus rules for the S4-plane

$$
\begin{array}{ccc}
\frac{\Gamma}{\square \perp, \diamond \Gamma} \mathrm{k}^{\prime} & \frac{A, \Gamma}{\square A, \diamond \Gamma, \diamond \perp, \ldots, \diamond \perp} \mathrm{k}^{\prime \prime} & \frac{\Gamma}{\square \perp, \diamond \Gamma, \diamond \perp, \ldots, \diamond \perp} \mathrm{k}^{\prime \prime \prime} \\
\frac{\Gamma, \diamond \Delta}{\square \perp, \diamond \Gamma, \diamond \Delta} 4_{\mathrm{k}}^{\prime} & \frac{A, \Gamma, \diamond \Delta}{\square A, \diamond \Gamma, \diamond \Delta, \diamond \perp, \ldots, \diamond \perp} 4_{\mathrm{k}}^{\prime \prime} & \frac{\Gamma, \diamond \Delta}{\square \perp, \diamond \Gamma, \diamond \Delta, \diamond \perp, \ldots, \diamond \perp} 4_{\mathrm{k}}^{\prime \prime \prime} \\
\frac{\Gamma}{\diamond \perp, \ldots, \diamond \perp, \diamond \Gamma} \mathrm{d}^{\prime} & \frac{\Gamma, \diamond \Delta}{\diamond \perp, \ldots, \diamond \perp, \diamond \Gamma, \diamond \Delta} 4_{\mathrm{dk}}^{\prime} &
\end{array}
$$

Fig. 3. Extended modal rules incorporating weakening on $\perp$.

$$
\begin{array}{cccc}
\frac{\Gamma\{A\}}{\Gamma\{A \vee B\}} \mathrm{w}_{\vee 1}^{\downarrow} & \frac{\Gamma\{A\}}{\Gamma\{B \vee A\}} \mathrm{w}_{\vee 2}^{\downarrow} & \frac{\Gamma\{\square \perp\}}{\Gamma\{\square A\}} \mathrm{w}_{\square}^{\downarrow} & \frac{\Gamma\{\diamond \perp\}}{\Gamma\{\diamond A\}} \mathrm{w}_{\diamond}^{\downarrow} \\
\frac{\Gamma\{A \vee A\}}{\Gamma\{A\}} \mathrm{c}^{\downarrow} & \frac{\Gamma\{A\}}{\Gamma\{\diamond A\}} \mathrm{t}^{\downarrow} & \frac{\Gamma\{\diamond \diamond A\}}{\Gamma\{\diamond A\}} 4^{\downarrow}
\end{array}
$$

Fig. 4. Deep inference rules for weakening, contraction, and the t- and 4-axioms

$$
\frac{\Gamma\{a \vee a\}}{\Gamma\{a\}} \mathrm{ac}^{\downarrow} \quad \frac{\Gamma\{(A \wedge B) \vee(C \wedge D)\}}{\Gamma\{(A \vee C) \wedge(B \vee D)\}} \mathrm{m} \quad \frac{\Gamma\{\square A \vee \square B\}}{\Gamma\{\square(A \vee B)\}} \mathrm{m}_{\square}^{\downarrow} \quad \frac{\Gamma\{\diamond A \vee \diamond B\}}{\Gamma\{\diamond(A \vee B)\}} \mathrm{m}_{\diamond}^{\downarrow}
$$

Fig. 5. The atomic contraction rule (where $a$ stands for any atom) and the medial rules

$$
\frac{\Gamma\{A \wedge(B \wedge C)\}}{\Gamma\{(A \wedge B) \wedge C\}} \equiv \frac{\Gamma\{A \vee(B \vee C)\}}{\Gamma\{(A \vee B) \vee C\}} \equiv \frac{\Gamma\{B \wedge A\}}{\Gamma\{A \wedge B\}} \equiv \frac{\Gamma\{B \vee A\}}{\Gamma\{A \vee B\}} \equiv
$$

Fig. 6. Equality rules

\begin{tabular}{l||c|c|c|c|c|c}
$\mathrm{X}$ & $\mathrm{K}$ & $\mathrm{KD}$ & $\mathrm{KT}$ & $\mathrm{K} 4$ & $\mathrm{KD} 4$ & $\mathrm{KT} 4=\mathrm{S} 4$ \\
\hline $\mathrm{X}^{\mathrm{seq}}$ & $\{\mathrm{k}\}$ & $\{\mathrm{k}, \mathrm{d}\}$ & $\{\mathrm{k}, \mathrm{t}\}$ & $\left\{\mathrm{k}, 4_{\mathrm{k}}\right\}$ & $\left\{\mathrm{k}, \mathrm{d}, 4_{\mathrm{k}}, 4_{\mathrm{dk}}\right\}$ & $\left\{\mathrm{k}, \mathrm{t}, 4_{\mathrm{k}}\right\}$ \\
$\mathrm{X}^{\mathrm{LL}}$ & $\left\{\mathrm{k}^{+}\right\}$ & $\left\{\mathrm{k}^{+}, \mathrm{d}^{+}\right\}$ & $\left\{\mathrm{k}^{+}\right\}$ & $\left\{\mathrm{k}^{+}\right\}$ & $\left\{\mathrm{k}^{+}, \mathrm{d}^{+}\right\}$ & $\left\{\mathrm{k}^{+}\right\}$ \\
$\mathrm{X}^{\downarrow}$ & $\varnothing$ & $\varnothing$ & $\left\{\mathrm{t}^{\downarrow}\right\}$ & $\left\{4^{\downarrow}\right\}$ & $\left\{4^{\downarrow}\right\}$ & $\left\{\mathrm{t}^{\downarrow}, 4^{\downarrow}\right\}$
\end{tabular}

Fig. 7. Rule sets from logics

We begin our presentation with the six modal logics in the S4-plane shown on the left of Figure 1. In Figure 2 we show the inference rules for the sequent systems for these logics. We use $\diamond \Gamma$ as abbreviation for $\diamond B_{1}, \ldots, \diamond B_{n}$ where $\Gamma=B_{1}, \ldots, B_{n}$. Then, Figure 3 shows variations of the modal rules that are 
needed to obtain our decomposition theorem (Theorem 2.2 below) that will play a crucial role in the proof of soundness and completeness for combinatorial proofs. We write $k^{+}$(resp. $\left.d^{+}, 4_{k}^{+}, 4_{d k}^{+}\right)$for any instance in $\left\{k, k^{\prime}, k^{\prime \prime}, k^{\prime \prime \prime}\right\}$ (resp. $\left\{d, d^{\prime}\right\},\left\{4_{k}, 4_{k}^{\prime}, 4_{k}^{\prime \prime}, 4_{k}^{\prime \prime \prime}\right\},\left\{4_{d k}, 4_{d k}^{\prime}\right\}$ ).

In this paper we also make use of some deep inference [12136] rules that are shown in Figure 4, where $\Gamma\{\}$ stands for a context, which is a sequent or a formula with a hole that takes the place of an atom. We write $\Gamma\{A\}$ when we replace the hole in $\Gamma\{\}$ by the formula $A$. We write $\mathrm{w}^{\downarrow}$ for the set $\left\{\mathrm{w}, \mathrm{w}_{\vee 1}^{\downarrow}, \mathrm{w}_{\vee 2}^{\downarrow}, \mathrm{w}_{\square}^{\downarrow}, \mathrm{w}_{\diamond}^{\downarrow}\right\}$.

For each $\mathrm{X}$ among the six logics $\mathrm{K}, \mathrm{KD}, \mathrm{KT}, \mathrm{K} 4, \mathrm{KD} 4$, and $\mathrm{KT} 4$, we define three sets $X^{\text {seq }}, X^{\mathrm{LL}}$, and $X^{\downarrow}$ of inference rules as shown in Figure 7 .

We now define the following sequent systems: $M L L=\{a x, \vee, \wedge\}$ and $L K=$ $M L L \cup\{w, c\}$; if $X$ is one of the six logics in the S4-plane, then MLL-X $=M L L \cup X^{\mathrm{LL}}$ and $L K-X=L K \cup X^{\text {seq. }}$. The following theorem is well-known [33].

Theorem 2.1 If $\mathrm{X} \in\{\mathrm{K}, \mathrm{KD}, \mathrm{KT}, \mathrm{K} 4, \mathrm{KD} 4, \mathrm{KT} 4\}$ then $\mathrm{LK}-\mathrm{X}$ is a sound and complete sequent system for the modal logic $\mathrm{X}$.

If $\Gamma$ is a sequent and $\mathrm{S}$ a sequent system, we write $\stackrel{\mathrm{S}}{\longleftarrow} \Gamma$ if there is a derivation of $\Gamma$ in $\mathrm{S}$. If $\mathrm{S}$ is s set of inference rules that all have exactly one premise, we can write $\Gamma^{\prime} \stackrel{\mathrm{S}}{\longleftarrow} \Gamma$ if there is a derivation from $\Gamma^{\prime}$ to $\Gamma$ using only rules from $\mathrm{S}$.

Theorem 2.2 Let $\mathrm{X} \in\{\mathrm{K}, \mathrm{KD}, \mathrm{KT}, \mathrm{K} 4, \mathrm{KD} 4, \mathrm{KT} 4\}$ and $\Gamma$ be a sequent. Then

$$
\stackrel{\mathrm{LK}-\mathrm{X}}{\longmapsto} \Gamma \quad \Longleftrightarrow \quad \stackrel{\mathrm{MLL}-\mathrm{X}}{\longmapsto} \Gamma^{\prime} \stackrel{\mathrm{X}^{\downarrow}}{\longmapsto} \Gamma^{\prime \prime} \stackrel{\mathrm{w}^{\downarrow}, c^{\downarrow}}{\longmapsto} \Gamma \text { for some } \Gamma^{\prime} \text { and } \Gamma^{\prime \prime} \text {. }
$$

Proof This is proved by a straightforward permutation argument. First, all instances of $w$ (resp. c) are replaced by instances of $w^{\downarrow}$ (resp. $\vee$ and $c^{\downarrow}$ ), and then all $w^{\downarrow}$ - and $c^{\downarrow}$-instances can be permuted down in the proof. Observe that this step introduces the rules shown in Figure 3 . Then, all $t$ instances are also $t^{\downarrow}$-instances, and all instances of $4_{k}^{+}$(resp. $4_{d k}^{+}$) are replaced by instances of $k^{+}$ (resp. $\mathrm{d}^{+}$) and $4^{\downarrow}$-instances. Then all $\mathrm{t}^{\downarrow}$ - and $4^{\downarrow}$-instances can be permuted down. Conversely, we can first permute the instances of $t \downarrow$ and $4 \downarrow$ up and then the instances of $w^{\downarrow}$ and $c^{\downarrow}$ until they are not deep anymore.

There are two reasons to use a deep contraction rule. The first is the decomposition theorem proved above, and the second is that deep contraction can be reduced to atomic form (shown on the left in Figure 5) via the so-called (deep) medial rules (shown on the right in Figure 5). We write $\mathrm{m}^{\downarrow}$ for the set $\left\{\mathrm{m}, \mathrm{m}_{\square}^{\downarrow}, \mathrm{m}_{\diamond}^{\downarrow}\right\}$. Additionally we make use of the equivalence rules shown in Figure6.

Theorem 2.3 Let $\Gamma^{\prime}$ and $\Gamma$ be sequents. Then

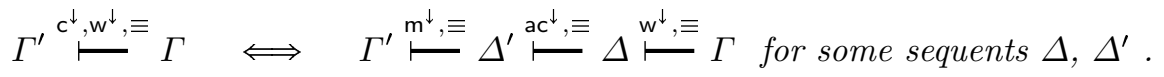

Proof For the case without modalities, this is a standard result in the calculus of structures, first proved in [6] (see also [27]). In the presence of the modalities, the proof is similar: For $\Rightarrow$ direction, we first reduce $c^{\downarrow}$ to ac $\downarrow$ using the medial 


$$
\begin{aligned}
& \frac{(A \vee B) \vee(A \vee B)}{A \vee B} \mathrm{c} \leadsto \frac{\frac{(A \vee B) \vee(A \vee B)}{\{A \vee A\} \vee\{B \vee B\}}}{A \vee B} \mathrm{c}^{\downarrow} \quad \frac{\square A \vee \square A}{\square A} \mathrm{c} \leadsto \frac{\frac{\square A \vee \square A}{\square A \vee A\}}}{\square A} \mathrm{c}_{\square}^{\downarrow} \\
& \frac{(A \wedge B) \vee(A \wedge B)}{A \wedge B} \mathrm{c} \leadsto \frac{\frac{(A \wedge B) \vee(A \wedge B)}{\{A \vee A\} \wedge\{B \vee B\}}}{A \vee B} \mathrm{~m}^{\downarrow} \quad \frac{\diamond A \vee \diamond A}{\diamond A} \mathrm{c} \leadsto \frac{\frac{\diamond A \vee \diamond A}{\diamond\{\vee A\}}}{\diamond A} \mathrm{c}_{\square}^{\downarrow}
\end{aligned}
$$

Fig. 8. Reducing contraction to atomic contraction via medial rules.

and equivalence rules, proceeding by induction on the contraction formula, as shown in Figure 8 . Note that a contraction on $\square \perp$ (resp. $\diamond \perp$ ) is already atomic. In the next step we permute the $w^{\downarrow}$ down, and finally we permute all instances of ac $\downarrow$ down. For the $\Leftarrow$ direction, observe that ac $\downarrow$ is already a special case of $c^{\downarrow}$ and that all rules in $\left\{m, m_{\square}^{\downarrow}, m_{\diamond}^{\downarrow}\right\}$ are derivable using $c^{\downarrow}$ and $w^{\downarrow}$.

\section{Relation Webs}

A directed graph $\mathcal{G}=\left\langle V_{\mathcal{G}}, \stackrel{\mathcal{G}}{\stackrel{\mathcal{A}}{ }\rangle}\right.$ is a set $V_{\mathcal{G}}$ of vertices equipped with a binary edge relation $\mathscr{\mathcal { G }} \subseteq V_{\mathcal{G}} \times V_{\mathcal{G}}$. We speak of an undirected graph $\mathcal{G}=\left\langle V_{\mathcal{G}}, \stackrel{\mathcal{G}}{=}\right\rangle$ if the edge relation $\mathcal{G} \subseteq V_{\mathcal{G}} \times V_{\mathcal{G}}$ is irreflexive and symmetric. A mixed graph is a

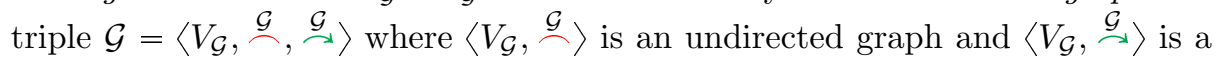
directed graph, such that $\stackrel{\mathcal{G}}{\lessgtr} \cap \mathscr{G}=\varnothing$ and $\mathscr{G}$ is irreflexive. From now on, we omit the index/superscript $\mathcal{G}$ when it is clear from the context. For two distinct vertices $v$ and $w$ in a mixed graph we use the following abbreviations:

$$
\begin{aligned}
& v \curvearrowleft w \Longleftrightarrow w \frown v \\
& v \curvearrowleft w \Longleftrightarrow v \frown w \text { or } v \curvearrowleft w \text { or } v \frown w \\
& v \smile w \Longleftrightarrow v \nrightarrow w \text { and } v \nprec w \text { and } v \nprec w
\end{aligned}
$$

Note that for any two vertices we have that $v \not w w$ iff $v \smile w$ or $v=w$. Furthermore, in a mixed graph, for any two vertices $v$ and $w$, exactly one of the following five statements is true:

$$
v=w \quad \text { or } \quad v \smile w \quad \text { or } \quad v \frown w \quad \text { or } \quad v \frown w \quad \text { or } \quad v \curvearrowleft w
$$

When drawing a graph we use $v-w$ for $v \frown w$, and $v \rightarrow w$ for $v \smile w$, and for $v \smile w$ we either use $v \cdots \cdots w$ or draw no edge at all.

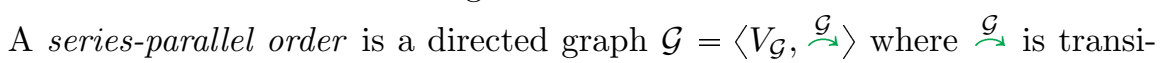
tive, irreflexive, and Z-free, i.e., $\mathcal{G}$ does not contain an induced subgraph of the shape shown on the left below:

Forbidden configurations for Z-freeness:

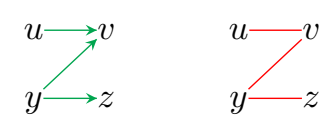


A cograph is an undirected graph that is Z-free, i.e., it does not contain an induced subgraph of the shape shown on the right above.

Definition 3.1 A relation web is a mixed graph $\mathcal{G}=\left\langle V_{\mathcal{G}}, \stackrel{\mathcal{G}}{\stackrel{\mathcal{G}}{4}}\right\rangle$ where $\left\langle V_{\mathcal{G}}, \stackrel{\mathcal{G}}{-}\right\rangle$ is a cograph and $\left\langle V_{\mathcal{G}}, \stackrel{\mathcal{G}}{\rightarrow}\right\rangle$ is a series-parallel order, and the following two configurations do not occur:

Forbidden configurations for relation webs:

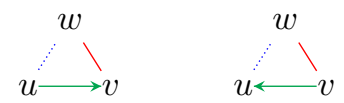

Observation 3.2 It is easy to see that in a relation web, the undirected graph determined by the relation $\smile$ (which is symmetric and irreflexive) is also a cograph.

Let $\mathcal{G}$ and $\mathcal{H}$ be two disjoint mixed graphs. We define the following operations:

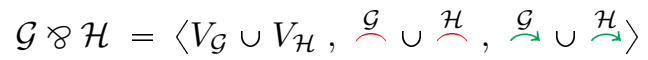

$$
\begin{aligned}
& \mathcal{G} \triangleleft \mathcal{H}=\left\langle V_{\mathcal{G}} \cup V_{\mathcal{H}}, \stackrel{\mathcal{G}}{\sim} \cup \stackrel{\mathcal{H}}{\rightarrow}, \stackrel{\mathcal{G}}{\sim} \cup \stackrel{\mathcal{H}}{\triangleleft} \cup\left\{(u, v) \mid u \in V_{\mathcal{G}}, v \in V_{\mathcal{H}}\right\}\right\rangle \\
& \mathcal{G} \otimes \mathcal{H}=\left\langle V_{\mathcal{G}} \cup V_{\mathcal{H}}, \stackrel{\mathcal{G}}{\sim} \cup \stackrel{\mathcal{H}}{=} \cup\left\{(u, v),(v, u) \mid u \in V_{\mathcal{G}}, v \in V_{\mathcal{H}}\right\}, \stackrel{\mathcal{G}}{\sim} \cup \stackrel{\mathcal{H}}{\sim}\right\rangle
\end{aligned}
$$

which can be visualized as follows:
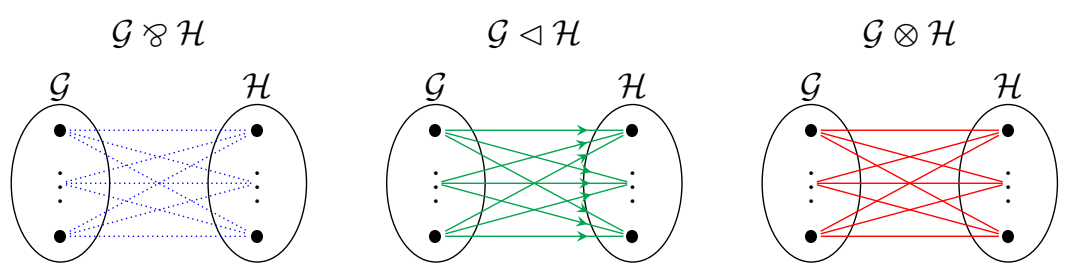

Theorem 3.3 A mixed graph is a relation web if and only if it can be constructed from single vertices using the three operations defined in (6) above.

Proof This follows from the corresponding results on cographs and series-parallel orders, e.g. [21]. A direct proof can be found in [12].

A relation web is labeled if all its vertices carry a label selected from a label set $\mathcal{L}$. We write $l(v)$ for the label of $v$. We are now defining for a formula $F$ the labeled relation web $\llbracket F \rrbracket$ where the label set $\mathcal{L}=\mathcal{A} \cup \overline{\mathcal{A}} \cup\{\square, \diamond\}$. We write $\varnothing$ for the empty graph and we use the notations $\bullet_{a}, \bullet_{\bar{a}}, \diamond, \square$ for the graph consisting of a single vertex that is labeled with $a, \bar{a}, \diamond, \square$, respectively.

$$
\begin{array}{llll}
\llbracket a \rrbracket=\bullet a & \llbracket A \wedge B \rrbracket=\llbracket A \rrbracket \otimes \llbracket B \rrbracket & \llbracket \square A \rrbracket=\square \triangleleft \llbracket A \rrbracket & \llbracket \square \perp \rrbracket=\square \\
\llbracket \bar{a} \rrbracket=\bullet \bar{a} & \llbracket A \vee B \rrbracket=\llbracket A \rrbracket \diamond \llbracket B \rrbracket & \llbracket \diamond A \rrbracket=\diamond \triangleleft \llbracket A \rrbracket & \llbracket \diamond \perp \rrbracket=\diamond
\end{array}
$$

For a sequent $\Gamma=A_{1}, \ldots, A_{n}$ we define $\llbracket \Gamma \rrbracket=\llbracket A_{1}, \ldots, A_{n} \rrbracket=\llbracket A_{1} \rrbracket 8 \cdots 8 \llbracket A_{n} \rrbracket$. 
Definition 3.4 A relation web $\mathcal{G}$ is modalic if for any vertices $u, v, w$ with $u \frown w$ and $v \triangleleft w$ we have $u \triangleleft v$ or $v \triangleleft u$ or $u=v$, i.e., $\mathcal{G}$ does not contain the two configurations below.

Forbidden configurations for modalic relation webs:

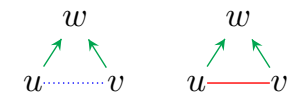

A labeled modalic relation web $\mathcal{G}$ is properly labeled if its label set is $\mathcal{L}=\mathcal{A} \cup$ $\overline{\mathcal{A}} \cup\{\square, \diamond\}$, such that whenever there are $v, w$ with $v \triangleleft w$ then $l(v) \in\{\square, \diamond\}$.

Theorem 3.5 A relation web is the translation of a modal formula if and only if it is modalic and properly labeled.

Proof If $\mathcal{G}=\llbracket F \rrbracket$ for some formula $F$, then the only vertices in $\mathcal{G}$ with outgoing $\triangle$-edge are the ones created in the encoding of a modal subformula and labeled with $\square$ or $\diamond$. If we have two distinct such vertices $u$ and $v$ with an $\lrcorner$-edge to some vertex $w$, then one of the corresponding modal operators is in the scope of the other and we have $u\lrcorner v$ or $v \triangleleft u$. The converse follows from Theorem 3.3 and the fact that the operation $\triangleleft$ in $(7)$ is associative. In fact, if $l(v)=$ (resp. $l(v)=\diamond$ ) and there is no $w$ such that $v \triangleleft w$ then we interpret the vertex $v$ as the subformula $\square \perp$ (resp. $\diamond \perp)$.

Proposition 3.6 For two formulas $F$ and $F^{\prime}$, we have $\llbracket F \rrbracket=\llbracket F^{\prime} \rrbracket$ iff $F$ and $F^{\prime}$ are equivalent modulo associativity and commutativity of $\wedge$ and $\vee$.

Proof By a straightforward induction, observing that the operations $\ngtr, \otimes$ and $\triangleleft$ in (7) are associative, and that 8 and $\otimes$ are also commutative.

Proposition 3.7 Given a set $V_{\mathcal{G}}$ and two binary relations $\stackrel{\mathcal{G}}{\mathcal{G}}, \subseteq V_{\mathcal{G}} \times V_{\mathcal{G}}$, it

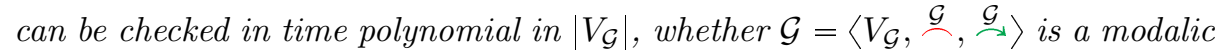
relation web.

Proof Checking the transitivity, irreflexivity, and symmetry for verifying that $\mathcal{G}$ is a mixed graph is trivially polynomial. Then, for checking the absence of the forbidden configurations in (4), (5), and (8) we can loop over all triples and quadruples of vertices, which is $O\left(\left|V_{\mathcal{G}}\right|^{4}\right)$.

\section{RGB-cographs and linear proofs for $\mathrm{K}$ and KD}

In this section we investigate when a modalic relation web does represent a proof. For this, we equip a relation web with a linking which is an equivalence class on its vertices. In the special case where each such equivalence class contains exactly two elements, and there are no $\frown$-edges, we have Retoré's RB-cographs 24 that with an additional correctness criterion correspond to proofs in MLL.

Here we generalize the notion of RB-cographs to the one of RGB-cographs and we give a correspondence with linear derivations in MLL-K and MLL-KD 4

\footnotetext{
4 The logics defined by these systems can be seen as the "linear logic variants" of the standard modal logics $\mathrm{K}$ and KD.
} 


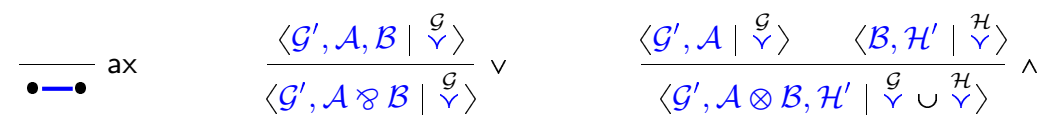

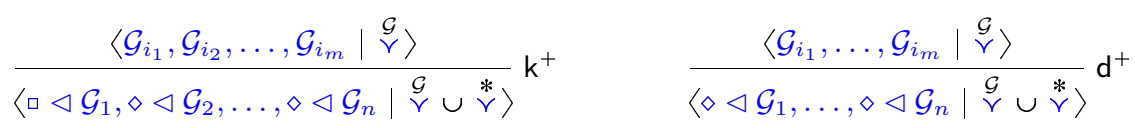

$$
\begin{aligned}
& \stackrel{*}{\vee}=\left\{(v, w) \mid v, w \in V^{\square} \uplus V^{\diamond} \text { and } v, w \notin V_{\mathcal{G}_{1}} \cup \cdots \cup V_{\mathcal{G}_{n}}\right\} \\
& i_{1}, \ldots, i_{m} \in\{1, \ldots, n\} \text { and pairwise distinct } \\
& \text { if } j \in\{1, \ldots, n\} \backslash\left\{i_{1}, \ldots, i_{m}\right\} \text { then } \mathcal{G}_{j}=\varnothing
\end{aligned}
$$

Fig. 9. Translating MLL-K and MLL-KD sequent proofs into RGB-cographs

Definition 4.1 An $R G B$-cograph is a tuple $\mathcal{G}=\left\langle V_{\mathcal{G}}, \stackrel{\mathcal{G}}{\underline{G}}, \stackrel{\mathcal{G}}{\mathcal{G}}, \stackrel{\mathcal{G}}{\text {, whe }}\right.$

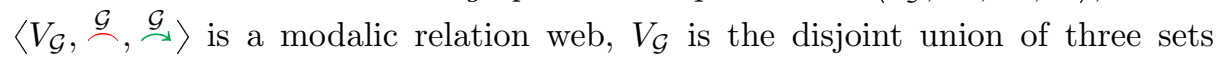
$V_{\mathcal{G}}^{\bullet} \uplus V_{\mathcal{G}}^{\mathrm{D}} \uplus V_{\mathcal{G}}^{\diamond}$, and $\stackrel{\mathcal{G}}{\gamma}$ is an equivalence relation, called the linking, such that

- if $v \in V_{\mathcal{G}}$ then for all $w \in V_{\mathcal{G}}$ we have $v \ngtr w$;

- if $v \vee w$ then either $v, w \in V^{\bullet}$ or $v, w \in V_{\mathcal{G}}^{\mathrm{D}} \uplus V_{\mathcal{G}}^{\diamond}$;

- if $v \in V_{\mathcal{G}}^{\bullet}$ then there is exactly one $w \in V_{\mathcal{G}}^{\bullet}$ with $v \vee w$ and $v \neq w$.

An equivalence class of $\underset{\mathcal{G}}{\vee}$ is called a link. The vertices in $V_{\mathcal{G}}^{\bullet}$ are called atomic vertices, and the vertices in $V_{\mathcal{G}}^{\square} \uplus V_{\mathcal{G}}^{\diamond}$ are called modalic vertices. An $R B$-cograph is an RGB-cograph $\mathcal{G}$ with $V_{\mathcal{G}}^{\mathrm{D}} \uplus V_{\mathcal{G}}^{\diamond}=\varnothing$.

The first condition in this definition says that if a vertex has an outgoing $\curvearrowright$ edge then it has to be in $V_{\mathcal{G}}^{\mathrm{D}} \uplus V_{\mathcal{G}}^{\diamond}$, the second condition says that vertices from $V_{\mathcal{G}}^{\bullet}$ and $V_{\mathcal{G}}^{\text {口}} \uplus V_{\mathcal{G}}^{\diamond}$ cannot be linked, and the third condition says that each link on $V_{\mathcal{G}}^{\bullet}$ has exactly two elements. In an RB-cograph 24] only the last condition makes sense since $\triangleleft$ is empty. When drawing an RGB-cograph we use bold (blue) edges $v-w$ when $v \neq w$ and $v \vee w$.

Figure 9 shows how proofs in MLL-K and MLL-KD are translated into RGBcographs. There, the notation $\left\langle\mathcal{G}_{1}, \mathcal{G}_{2}, \ldots, \mathcal{G}_{n} \mid \stackrel{\mathcal{G}}{\gamma}\right\rangle$ is used to denote the RGBcograph whose underlying relation web is $\mathcal{G}=\mathcal{G}_{1} \ngtr \mathcal{G}_{2} \ngtr \cdots \ngtr \mathcal{G}_{n}$ and whose linking is $\mathcal{G}$. The ax-rule simply produces a graph with two vertices that are linked, and $\frown$ and $\curvearrowright$ being empty. In the $\vee$-rule, premise and conclusion are the same RGB-cograph. In the $\wedge$-rule, the linking in the conclusion is the union of the linkings in the premises. These three rules behave exactly the same as in proof nets for MLL. More interesting are the rules $\mathrm{k}^{+}$and $\mathrm{d}^{+}$, where the linking of the conclusion is the linking of the premise together with an additional equivalence class containing all the new $\square$ - and $\diamond$-vertices. The purpose of this section is to give a combinatorial characterization of the RGB-cographs that can be obtained via this sequent calculus translation.

Definition 4.2 An alternating elementary path (a-path) of length $n$ in an RGB-cograph is a sequence of pairwise distinct vertices $x_{0}, x_{1}, \ldots, x_{n}$ such that we have either $x_{0} \curlyvee x_{1} R_{1} x_{2} \curlyvee x_{3} R_{3} x_{4} \cdots x_{n}$ or $x_{0} R_{0} x_{1} \curlyvee x_{2} R_{2} x_{3} \vee x_{4} \cdots x_{n}$ with 
$R_{i} \in\{\frown, \frown\}$, i.e., an $æ$-path is an elementary path whose edges are alternating in $\checkmark$ and $\frown \cup \frown$. A chord in an æ-path is an edge $x_{i} \frown x_{j}$ or $x_{i} \frown x_{j}$ for $i, j \in\{0, \ldots, n\}$ and $i+2 \leqslant j$. A chordless ae-path is an $æ$-path without chord. An $x$-cycle is an $æ$-path of even length such that $x_{0}=x_{n}$. An RGB-cograph $\mathcal{G}$ is $\mathfrak{a}$-connected if any two vertices are conneced by a chordless æ-path, and $\mathcal{G}$ is $a$-acyclic if it contains no chordless æ-cycle.

Definition 4.3 An RGB-cograph $\mathcal{G}$ is $\mathrm{K}$-correct (or $\left\{\mathrm{k}^{+}\right\}$-correct) if

1. $\mathcal{G}$ is $æ$-connected and $æ$-acyclic;

2. $V_{\mathcal{G}} \neq \varnothing$ and every $\checkmark$-equivalence class in $V^{\square} \uplus V^{\diamond}$ contains at least one vertex $v$ such that there is a vertex $w \in V^{\bullet}$ with $v \triangleleft w$;

3. if $w \stackrel{\mathcal{G}}{\sim} v$ and $v \vee v^{\prime}$, then there is $w^{\prime} \vee w$ such that $w^{\prime} \mathcal{G} v^{\prime}$; and

4. every $\curlyvee$-equivalence class in $V^{\mathrm{D}} \uplus V^{\diamond}$ contains exactly one vertex $v \in V^{\mathrm{D}}$.

We say that $\mathcal{G}$ is $\mathrm{KD}$-correct (or $\left\{\mathrm{k}^{+}, \mathrm{d}^{+}\right\}$-correct) if Condition 4 is replaced by:

4. every $\checkmark$-equivalence class in $V^{\square} \uplus V^{\diamond}$ contains at most one vertex $v \in V^{\square}$.

Theorem 4.4 Let $\mathrm{X} \in\{\mathrm{K}, \mathrm{KD}\}$. An RGB-cograph $\mathcal{G}$ is the translation of an MLL-X sequent proof iff it is $\mathrm{X}^{\mathrm{LL}}$-correct.

Proof (Sketch) For the left-to-right direction, observe that all rules in Figure 9 preserve correctness. For the right-to-left direction, we will reuse the MLL sequentialization result for RB-cographs [24]. For this we will define for an RGB-cograph $\mathcal{G}$ an RB-cograph $\partial(\mathcal{G})$ that is æ-connected and æ-acyclic if and only if $\mathcal{G}$ is. We define a vertex set $V^{*}=\left\{v^{\prime}, \bar{v}^{\prime} \mid v \in V_{\mathcal{G}}^{\mathrm{D}} \uplus V_{\mathcal{G}}^{\diamond}\right\}$ and let $V_{\partial(\mathcal{G})}=V_{\mathcal{G}} \uplus V^{*}$, i.e., we take the atomic vertices of $\mathcal{G}$, and each modalic vertex is replaced by a dual pair of atomic vertices, that are linked by $\checkmark$. Then we use Theorem 3.3 and Proposition 3.6 so that we can write $\mathcal{G}$ and $\partial(\mathcal{G})$ as BV-formulas [12]. Let $v_{1}, \ldots, v_{n} \in V_{\mathcal{G}}^{\mathrm{a}} \uplus V_{\mathcal{G}}^{\diamond}$ form an $\stackrel{\mathcal{G}}{\gamma}$-equivalence class. Then the formula for $\mathcal{G}$ is of shape $F\left\{v_{1} \triangleleft B_{1}\right\} \cdots\left\{v_{n} \triangleleft B_{n}\right\}$ for some $n$-ary context $F\{\} \cdots\{\}$ (because $\mathcal{G}$ is modalic). We transform $F\left\{v_{1} \triangleleft B_{1}\right\} \cdots\left\{v_{n} \triangleleft B_{n}\right\}$ into $\left(\bar{v}_{1}^{\prime} \otimes \cdots \otimes \bar{v}_{n}^{\prime} \otimes \partial\left(B_{1} \ngtr \cdots \otimes B_{n}\right)\right) \ngtr \partial\left(F\left\{v_{1}^{\prime}\right\} \cdots\left\{v_{n}^{\prime}\right\}\right)$ and proceed inductively

for all $\mathcal{G}$-equivalence classes. From Retoré's proof 24 we get an MLL sequentialization for $\partial(\mathcal{G})$, which we then transform back into an MLL-K or MLL-KD sequent proof for $\mathcal{G}$.

\section{Skew Fibrations}

Definition 5.1 Let $\mathcal{G}$ and $\mathcal{H}$ be mixed graphs. A skew fibration $f: \mathcal{G} \rightarrow \mathcal{H}$ is a function from $V_{\mathcal{G}}$ to $V_{\mathcal{H}}$ that preserves $\frown$ and $\frown$, i.e.,

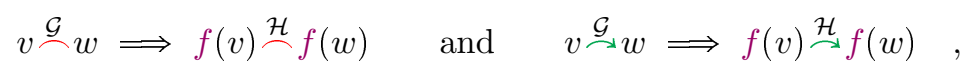

and has the skew lifting property, i.e.,

for every $v \in V_{\mathcal{G}}$ and $w \in V_{\mathcal{H}}$ and $R \in\{\frown, \frown\}$ with $w R_{\mathcal{H}} f(v)$, there is a $u \in V_{\mathcal{G}}$ such that $u R_{\mathcal{G}} v$ and $w \stackrel{\mathcal{H}}{\leftarrow} f(u)$ and $w^{\stackrel{H}{f}} f(u)$. 

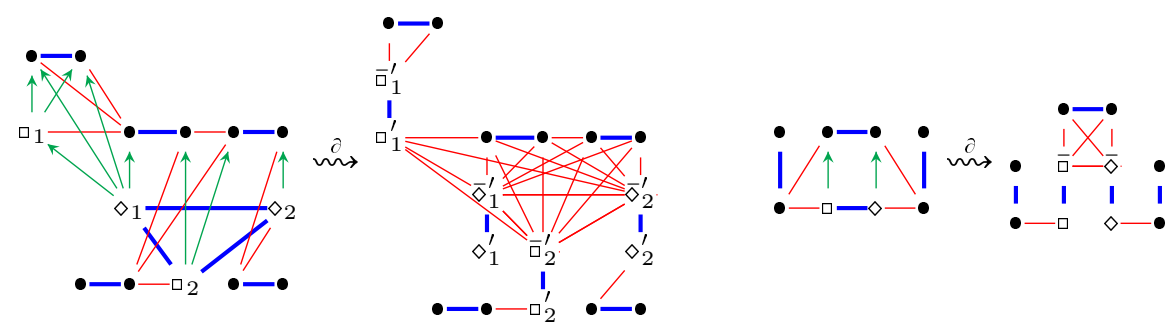

Fig. 10. The RGB-cographs for $F_{1}=\bar{d} \vee(d \wedge \square(\bar{b} \wedge c) \vee \bar{e} \vee(e \wedge \diamond \bar{c}) \vee \diamond(b \wedge \square(a \vee \bar{a})))$ and $F_{2}=b \vee(\bar{b} \wedge \square a) \vee(\diamond \bar{a} \wedge c) \vee \bar{c}$, and the corresponding RB-cographs $\partial\left(F_{1}\right)$ and $\partial\left(F_{2}\right)$.

A skew fibration $f: \mathcal{G} \rightarrow \mathcal{H}$ is modalic if it satisfies the following condition:

$$
\begin{aligned}
& \text { if } u \mathscr{G} v \text { and } f(u) \stackrel{\mathcal{H}}{\stackrel{\mathcal{G}}{ }} f(v) \text {, then there is a } w \in V_{\mathcal{G}} \text { such that } \\
& w \mathscr{\mathcal { G }} v \text { and } f(u)=f(w) \text {, or } u \mathscr{\mathcal { G }} w \text { and } f(v)=f(w) .
\end{aligned}
$$

The main purpose of this definition is Theorem 5.2 which says that skew fibrations are precisely the contraction-weakening maps. This is crucial for the soundness and completeness of combinatorial proofs, to be defined in the next section.

Theorem 5.2 There is a modalic skew fibration $f: \llbracket \Gamma^{\prime} \rrbracket \rightarrow \llbracket \Gamma \rrbracket$ iff $\Gamma^{\prime^{c^{\downarrow}}, w^{\downarrow}, \equiv} \Gamma$.

Proof (Sketch) To prove this theorem, we proceed via Theorem 2.3 and make heavy use of results from [27] and the fact that $\Gamma^{\prime} \stackrel{\mathrm{m}^{\downarrow}, \equiv}{\longmapsto} \Gamma$ iff there is a surjective modalic skew fibration $f: \llbracket \Gamma^{\prime} \rrbracket \rightarrow \llbracket \Gamma \rrbracket$ that is bijective on atomic vertices, which is a variant of [27, Theorem 5.1] and proved in a similar way. Then we can characterize derivations $\stackrel{\mathrm{ac}^{\downarrow}, \equiv}{\longmapsto}$ and $\stackrel{\mathrm{w}^{\downarrow}, \equiv}{\longmapsto}$ as in [27, Proposition 7.6], so that we can apply Theorem 2.3 (See also [2] and [4]).

\section{Combinatorial proofs for the modal logics $K$ and $D$}

Definition 6.1 A map $f: \mathcal{G} \rightarrow \mathcal{F}$ from an RGB-cograph $\mathcal{G}$ to a modalic and properly labeled relation web $\mathcal{F}$ is allegiant if the following conditions hold:

- if $v, w \in V_{\dot{\mathcal{G}}}$ and $v \stackrel{\mathcal{G}}{\vee} w$ then $f(v)$ and $f(w)$ are labeled by dual atoms;

- if $v \in V_{C}^{\text {口 }}$ then $l(f(v))=\square$;

- if $v \in V_{\mathcal{G}}^{\diamond}$ then $l(f(v))=\diamond$;

Definition 6.2 For $\mathrm{X} \in\{\mathrm{K}, \mathrm{KD}\}$, an $\mathrm{X}$-combinatorial proof of a sequent $\Gamma$ is an allegiant skew-fibration $f: \mathcal{G} \rightarrow \llbracket \Gamma \rrbracket$ from an X-correct RGB-cograph $\mathcal{G}$ to the relation web of $\Gamma$.

The size $|f|$ of a combinatorial proof $f: \mathcal{G} \rightarrow \llbracket \Gamma \rrbracket$ is $\left|V_{\mathcal{G}}\right|+|\Gamma|$, where $|\Gamma|$ is the number of symbols in $\Gamma$, and the size $|\pi|$ of a sequent proof $\pi$ is the number of symbols in $\pi$. 
Theorem 6.3 (Completeness) Let $\mathrm{X} \in\{\mathrm{K}, \mathrm{KD}\}$. If $\stackrel{\mathrm{LK}-\mathrm{X}}{\longmapsto} \Gamma$ then there is an $\mathrm{X}$-combinatorial proof $f: \mathcal{G} \rightarrow \llbracket \Gamma \rrbracket$. Furthermore, the sizes of the sequent proof and the combinatorial proof differ only by a polynomial factor.

Proof Let $\pi$ be a proof of $\Gamma$ in LK-X. By Theorem 2.2, $\pi$ can be rewritten as $\stackrel{\text { MLL-X }}{\longleftarrow} \Gamma^{\prime} \stackrel{w^{\downarrow}, c^{\downarrow}}{\longleftarrow} \Gamma$ for some $\Gamma^{\prime}$. By Theorem 4.4 we have an X-correct RGBcograph $\mathcal{G}$ whose underlying relation web is $\llbracket \Gamma^{\prime} \rrbracket$. By Theorem 5.2 we have a skew-fibration $f: \llbracket \Gamma^{\prime} \rrbracket \rightarrow \llbracket \Gamma \rrbracket$, and therefore also $f: \mathcal{G} \rightarrow \llbracket \Gamma \rrbracket$, which is allegiant by construction. The size restrictions follow immediately: sequent proof and combinatorial proof are bound by the number of $\mathrm{ax}, \mathrm{k}^{+}$, and $\mathrm{d}^{+}$instances.

Theorem 6.4 (Soundness) Let $\mathrm{X} \in\{\mathrm{K}, \mathrm{KD}\}$, and let $f: \mathcal{G} \rightarrow \llbracket A \rrbracket$ be an $\mathrm{X}$ combinatorial proof. Then $A$ is a theorem in the modal logic $\mathrm{X}$.

Proof We have an MLL-X proof of a formula $A^{\prime}$ with $\llbracket A^{\prime} \rrbracket=\mathcal{G}$. Hence $A^{\prime}$ is a theorem of X. By Theorem 5.2 we have a derivation $A^{\prime} \stackrel{c^{\downarrow}, w^{\downarrow}, \equiv}{\longmapsto} A$ in which all inferences are sound for $\mathrm{X}$, we can conclude that $A$ is also a theorem of $\mathrm{X}$.

Theorem 6.5 Let $\Gamma$ be a sequent, $\mathcal{G}$ be a mixed graph together with a linking, and let $f$ be a map from $\mathcal{G}$ to $\llbracket \Gamma \rrbracket$. It can be decided in polynomial time in $\left|V_{\mathcal{G}}\right|+|\Gamma|$ whether $f: \mathcal{G} \rightarrow \llbracket \Gamma \rrbracket$ is an $\mathrm{X}$-combinatorial proof for $\mathrm{X} \in\{\mathrm{K}, \mathrm{KD}\}$.

Proof All necessary properties (forbidden configurations (4), (5), (8) for $\mathcal{G}$ being a modalic relation web, $\mathrm{X}$-correctness conditions in Definition 4.3, preservation of $\frown$ and $\frown(9)$ and skew lifting (10) can be checked in polynomial time.

These three results, together with Theorem 2.1, imply that $\mathrm{X}$-combinatorial proofs (for $\mathrm{X}=\mathrm{K}$ and $\mathrm{X}=\mathrm{KD}$ ) form a sound and complete proof system (in the sense of [9]) for the modal logic $X$. In the remaining sections of this paper we extend this result to all logics in the S4-tesseract.

\section{Combinatorial proofs for the logics in the S4-plane}

We call two vertices $v$ and $w$ in a relation web $\mathcal{G}$ clones if for all $u$ with $u \neq v$ and $u \neq w$ we have $u R v$ iff $u R w$ for all $R \in\{\frown, \frown, \curvearrowleft, \smile\}$. If $v=w$ then they are trivially clones.

Definition 7.1 Let $\mathcal{G}$ and $\mathcal{H}$ be modalic and properly labeled relation webs. A map $f: \mathcal{G} \rightarrow \mathcal{H}$ is a $\left\{4^{\downarrow}, \mathrm{t}^{\downarrow}\right\}-$ map if the following conditions are fulfilled:

- if $f(v)=f(w)$ then $v$ and $w$ are clones in $\mathcal{G}$, and if also $v \neq w$ then $v \stackrel{\mathcal{G}}{w} w$ and $l(f(v))=l(f(w))=\diamond$;

- if $f(v) \neq f(w)$ then $v R_{\mathcal{G}} w$ implies $f(v) R_{\mathcal{H}} f(w)$ for any $R \in\{\frown, \frown, \curvearrowleft, \smile\}$;

- if $v \in V_{\mathcal{H}}$ is not in the image of $f$ then $l(v)=\diamond$ and $v \triangleleft w$ for some $w \in V_{\mathcal{H}}$. A $\left\{4^{\downarrow}, \mathrm{t}^{\downarrow}\right\}$-map is a $\left\{\mathrm{t}^{\downarrow}\right\}-$-map if it is injective, and a $\left\{4^{\downarrow}\right\}$-map if it is surjective.

Proposition 7.2 The composition of $\left\{4^{\downarrow}, \mathrm{t}^{\downarrow}\right\}$-maps is a $\left\{4^{\downarrow}, \mathrm{t}^{\downarrow}\right\}$-map, and every $\left\{4^{\downarrow}, \mathrm{t}^{\downarrow}\right\}$-map can be written as a composition of $a\{4 \downarrow\}$-map and a $\{\mathrm{t} \downarrow\}$-map. 
Lemma 7.3 For all sequents $\Gamma$ and $\Gamma^{\prime}$, we have:

$-\Gamma^{\prime} \stackrel{4^{\downarrow}, \mathrm{t}^{\downarrow}, \equiv}{\longmapsto} \Gamma$ iff there is $a\left\{4^{\downarrow}, \mathrm{t}^{\downarrow}\right\}-$ map $f: \llbracket \Gamma^{\prime} \rrbracket \rightarrow \llbracket \Gamma \rrbracket$;

$-\Gamma^{\prime} \stackrel{4^{\downarrow}, \equiv}{\longmapsto} \Gamma$ iff there is a $\left\{4^{\downarrow}\right\}$-map $f: \llbracket \Gamma^{\prime} \rrbracket \rightarrow \llbracket \Gamma \rrbracket$;

$-\Gamma^{\prime} \stackrel{\mathrm{t}^{\downarrow}}{\longmapsto} \Gamma$ iff there is a $\left\{\mathrm{t}^{\downarrow}\right\}$-map $f: \llbracket \Gamma^{\prime} \rrbracket \rightarrow \llbracket \Gamma \rrbracket$;

Proof The second and the third statement follow immediately from the definitions, and for the first statement, observe that $\Gamma^{\prime} \stackrel{4^{\downarrow}, \mathrm{t}^{\downarrow}, \equiv}{\longmapsto} \Gamma$ iff $\Gamma^{\prime} \stackrel{4^{\downarrow}, \equiv}{\longmapsto} \Gamma^{\prime \prime} \stackrel{\mathrm{t}^{\downarrow}, \equiv}{\longmapsto} \Gamma$, and apply Proposition 7.2

Definition 7.4 Let $\mathrm{X} \in\{\mathrm{K}, \mathrm{KD}, \mathrm{KT}, \mathrm{K} 4, \mathrm{KD} 4, \mathrm{KT} 4\}$. A map $f: \mathcal{G} \rightarrow \mathcal{H}$ is an $X^{\downarrow}$-skew fibration if $f=f^{\prime \prime} \circ f^{\prime}$ for some $f^{\prime}: \mathcal{G} \rightarrow \mathcal{G}^{\prime}$ and $f^{\prime \prime}: \mathcal{G}^{\prime} \rightarrow \mathcal{H}$, where $f^{\prime}$ is an $X^{\downarrow}$-map and $f^{\prime \prime}$ is a modalic skew fibration (if $X^{\downarrow}=\varnothing$ then $f^{\prime}$ is the identity).

Proposition 7.5 Given $f: \mathcal{G} \rightarrow \mathcal{H}$ and $\mathrm{X}^{\downarrow} \subseteq\left\{\mathrm{t}^{\downarrow}, 4^{\downarrow}\right\}$, it can be decided in time polynomial in $|\mathcal{G}|+|\mathcal{H}|$ whether $f$ is an $X^{\downarrow}$-skew fibration.

Below are three examples, a $\left\{t^{\downarrow}\right\}$-skew fibration, a $\left\{4^{\downarrow}\right\}$-skew fibration, and a $\left\{4^{\downarrow}, t^{\downarrow}\right\}$-skew fibration:
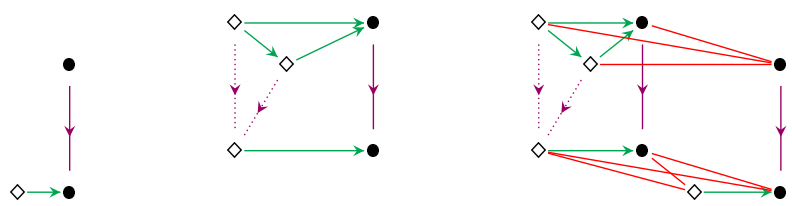

We can now easily generalize Theorem 5.2

Theorem 7.6 Let $\mathrm{X}^{\downarrow} \subseteq\left\{\mathrm{t}^{\downarrow}, 4^{\downarrow}\right\}$. There is a derivation $\Gamma^{\prime} \stackrel{\mathrm{X}^{\downarrow}, \equiv}{\longmapsto} \Gamma^{\prime \prime} \stackrel{\mathrm{c}^{\downarrow}, \mathrm{w}^{\downarrow}, \equiv}{\longmapsto} \Gamma$ iff there is an $\mathrm{X}^{\downarrow}$-skew fibration $f: \llbracket \Gamma^{\prime} \rrbracket \rightarrow \llbracket \Gamma \rrbracket$.

Proof This follows immediately from Definition 7.4, Lemma 7.3 and Theorem 5.2

Definition 7.7 For $\mathrm{X} \in\{\mathrm{K}, \mathrm{KD}, \mathrm{KT}, \mathrm{K} 4, \mathrm{KD} 4, \mathrm{KT} 4\}$, an $\mathrm{X}$-combinatorial proof of a sequent $\Gamma$ is an allegiant $\mathrm{X}^{\downarrow}$-skew-fibration $f: \mathcal{G} \rightarrow \llbracket \Gamma \rrbracket$ from an $\mathrm{X}^{\mathrm{LL}}$-correct RGB-cograph $\mathcal{G}$ to the relation web of $\Gamma$.

With this definition, it now follows immediately from Proposition 7.5. Theorem 7.6 and Theorem 2.2 that Theorems 6.3, 6.4 and 6.5 hold for all $\mathrm{X} \in$ $\{\mathrm{K}, \mathrm{KD}, \mathrm{KT}, \mathrm{K} 4, \mathrm{KD} 4, \mathrm{KT} 4\}$. 


$$
\begin{array}{cccccc}
\frac{A, B}{\square A, \diamond B} \mathrm{~m} & \frac{A, B}{\diamond A, \diamond B} \mathrm{~d}_{\mathrm{m}} & \frac{A, \diamond B}{\square A, \diamond B} 4_{\mathrm{m}} & \frac{A, \diamond B}{\diamond A, \diamond B} 4_{\mathrm{dm}} & \frac{A}{\square A} \mathrm{n} & \frac{A}{\diamond A} \mathrm{p} \\
\frac{A, \Gamma}{\square A, \diamond \Gamma} \mathrm{k}_{\mathrm{c}} & \frac{A, \Gamma}{\diamond A, \diamond \Gamma} \mathrm{d}_{\mathrm{c}} & \frac{A, \Gamma, \diamond \Delta}{\square A, \diamond \Gamma, \diamond \Delta} 4_{\mathrm{c}} & \frac{A, \Gamma, \diamond \Delta}{\diamond A, \diamond \Gamma, \diamond \Delta} 4_{\mathrm{dc}} & |\Gamma, \Delta|>0
\end{array}
$$

Fig. 11. Sequent calculus rules for S4-tesseract logics.

$$
\begin{aligned}
& \frac{B}{\square \perp, \diamond B} \mathrm{~m}^{\prime} \quad \frac{A}{\square A, \diamond \perp} \mathrm{m}^{\prime \prime} \quad \frac{A}{\diamond \perp, \diamond A} \mathrm{~d}_{\mathrm{m}}^{\prime} \quad \frac{\diamond A}{\square \perp, \diamond A} 4_{\mathrm{m}}^{\prime} \quad \frac{\diamond A}{\diamond \perp, \diamond A} 4_{\mathrm{dm}}^{\prime} \\
& \frac{\Gamma}{\square \perp, \diamond \Gamma} \mathrm{k}_{c}^{\prime} \quad \frac{A, \Gamma}{\square A, \diamond \Gamma, \diamond \perp, \ldots, \diamond \perp} \mathrm{k}_{c}^{\prime \prime} \quad \frac{\Gamma}{\square \perp, \diamond \Gamma, \diamond \perp, \ldots, \diamond \perp} \mathrm{k}_{c}^{\prime \prime \prime} \\
& \frac{\Gamma, \diamond \Delta}{\square,, \diamond \Gamma, \diamond \Delta} 4_{c}^{\prime} \quad \frac{A, \Gamma, \diamond \Delta}{\square A, \diamond \Gamma, \diamond \Delta, \diamond \perp, \ldots, \diamond \perp} 4_{c}^{\prime \prime} \quad \frac{\Gamma, \diamond \Delta}{\square \perp, \diamond \Gamma, \diamond \Delta, \diamond \perp, \ldots, \diamond \perp} 4_{c}^{\prime \prime \prime} \\
& \frac{\Gamma}{\diamond \perp, \ldots, \diamond \perp, \diamond \Gamma} \mathrm{d}_{\mathrm{c}}^{\prime} \quad \frac{\Gamma, \diamond \Delta}{\diamond \perp, \ldots, \diamond \perp, \diamond \Gamma, \diamond \Delta} 4_{\mathrm{dc}^{\prime}} \quad|\Gamma, \Delta|>0 \\
& \mathrm{~m}^{+}=\left\{\mathrm{m}, \mathrm{m}^{\prime}, \mathrm{m}^{\prime \prime}\right\} \quad \mathrm{d}_{\mathrm{m}}^{+}=\left\{\mathrm{d}_{\mathrm{m}}, \mathrm{d}_{\mathrm{m}}^{\prime}\right\} \quad 4_{\mathrm{m}}^{+}=\left\{4_{\mathrm{m}}, 4_{\mathrm{m}}^{\prime}\right\} \quad 4_{\mathrm{dm}}^{+}=\left\{4_{\mathrm{dm}}, 4_{\mathrm{dm}}^{\prime}\right\} \\
& k_{c}^{+}=\left\{k_{c}, k_{c}^{\prime}, k_{c}^{\prime \prime}, k_{c}^{\prime \prime \prime}\right\} \quad d_{c}^{+}=\left\{d_{c}, d_{c}^{\prime}\right\} \quad 4_{c}^{+}=\left\{4_{c}, 4_{c}^{\prime}, 4_{c}^{\prime \prime}, 4_{c}^{\prime \prime \prime}\right\} \quad 4_{d c}^{+}=\left\{4_{d c}, 4_{d c}^{\prime}\right\}
\end{aligned}
$$

Fig. 12. Extended non-normal modal logic rules incorporating weakening on $\perp$.

\section{Non-normal modal logics}

In this section, we show how to extend the results for the logics of the S4-plane to the non-normal modal logics of the S4-tesseract $t^{5}$ in Figure 1 . Figure 11 shows the additional sequent rules that are needed for these logics, Figure 12 shows the variations of these rules that are needed for obtaining the decomposition in Theorem 2.2, and Figure 13 shows the extension of the table in Figure 7 , defining a sound and complete sequent system for each logic. We state here only the Theorem 8.1 below, and refer the reader to the exposition in [19] for more details, references, and proofs.

Theorem 8.1 For all modal logics $\mathrm{X}$ of the S4-tesseract, LK-X is a sound and complete sequent system for the modal logic $\mathrm{X}$.

For our purpose, the most important observation is that the Decomposition Theorem 2.2 holds for all of these logics. This means that for defining combinatorial proofs for these logics, it suffices to define for RGB-cographs what it means to be $\mathrm{X}^{\mathrm{LL}}$-correct.

\footnotetext{
${ }^{5}$ Observe that all the logics in the S4-tesseract are monotone. In fact, our methods can not be applied in presence of the rule $\frac{A, \bar{B} \quad \bar{A}, B}{\square A, \diamond B} \mathrm{E}$. We therefore have to leave the investigation of combinatorial proofs for non-monotonic non-normal modal logics as an open problem for future research.
} 


\begin{tabular}{|c|c|c|c|c|c|c|c|c|c|c|}
\hline$X$ & $\mathrm{M}$ & M4 & & ИP & \multicolumn{2}{|c|}{ MP4 } & MD & MD4 & MT & MT4 \\
\hline$\overline{X^{\text {seq }}}$ & $\{m\}$ & $\left\{m, 4_{m}\right\}$ & & $p, p\}$ & $\{m, p$ &, $4 m$ & $\left\{\mathrm{~m}, \mathrm{~d}_{\mathrm{m}}\right\}$ & $\left\{\mathrm{m}, \mathrm{d}_{\mathrm{m}}, 4_{\mathrm{m}}\right\}$ & $\{m, t\}$ & $\overline{\left\{m, t, 4_{m}\right\}}$ \\
\hline$X^{L L}$ & $\left\{m^{+}\right\} \mid\{r$ & $\left\{\mathrm{m}^{+}, 4_{\mathrm{m}}^{+}\right\}$ & $\left\{m^{+}\right.$ & $+, p\}$ & $\left\{\mathrm{m}^{+}\right.$ & p $\}$ & $\left\{\mathrm{m}^{+}, \mathrm{d}_{\mathrm{m}}^{+}\right\}$ & \}$\left\{\mathrm{m}^{+}, \mathrm{d}_{\mathrm{m}}^{+}\right\}$ & $\left\{m^{+}\right\}$ & $\left\{m^{+}\right\}$ \\
\hline$X^{\downarrow}$ & $\varnothing$ & $\left\{4^{\downarrow}\right\}$ & & $\varnothing$ & $\{4$ & & $\varnothing$ & $\left\{4^{\downarrow}\right\}$ & $\left\{t^{\downarrow}\right\}$ & $\left\{\mathrm{t}^{\downarrow}, 4^{\downarrow}\right\}$ \\
\hline X & $\mathrm{N}$ & N4 & & NP & & & NP4 & ND & $\mathrm{NL}$ & D4 \\
\hline$\overline{X^{\text {seq }}}$ & $\{m, n\}$ & $\{m, n, 4$ & & $\{m, n$ & $, p\}$ & $\{m, 1$ & $\left.\mathrm{n}, \mathrm{p}, 4_{\mathrm{m}}\right\}$ & $\left\{\mathrm{m}, \mathrm{n}, \mathrm{d}_{\mathrm{m}}\right\}$ & $\mathrm{m}, \mathrm{n}, \mathrm{d}_{\mathrm{m}}$ & $\left., 4_{\mathrm{m}}, 4_{\mathrm{dm}}\right\}$ \\
\hline$X^{L L}$ & $\left\{\mathrm{~m}^{+}, \mathrm{n}\right\}$ & \}$\left\{\mathrm{m}^{+}, \mathrm{n}\right.$ & & $\left\{m^{+}, r\right.$ & $n, p\}$ & $s m$ & $+, n, p\}$ & $\left\{\mathrm{m}^{+}, \mathrm{n}, \mathrm{d}_{\mathrm{m}}^{+}\right\}$ & $\left\{\mathrm{m}^{+}\right.$, & $\left.\mathrm{n}, \mathrm{d}_{\mathrm{m}}^{+}\right\}$ \\
\hline$X^{\downarrow}$ & $\varnothing$ & $\left\{4^{\downarrow}\right\}$ & & $\varnothing$ & & & $\left\{4^{\downarrow}\right\}$ & $\varnothing$ & & \} \\
\hline & NT & NT4 & & C & $\mathrm{C}$ & & $C D$ & CD4 & CT & CT4 \\
\hline $\mathrm{K}^{\mathrm{seq}}$ & $\{\mathrm{m}, \mathrm{n}, \mathrm{t}\}$ & $\{\mathrm{m}, \mathrm{n}, \mathrm{t}$ & $\left.44_{m}\right\}$ & $\left\{\mathrm{k}_{c}\right\}$ & $\left\{\mathrm{k}_{\mathrm{c}}\right.$, & $\left.44_{c}\right\}$ & $\left\{\mathrm{k}_{\mathrm{c}}, \mathrm{d}_{\mathrm{c}}\right\}\{$ & $\left\{\mathrm{k}_{\mathrm{c}}, \mathrm{d}_{\mathrm{c}}, 4_{\mathrm{c}}, 4_{\mathrm{dc}}\right\}$ & \}$\left\{\mathrm{k}_{\mathrm{c}}, \mathrm{t}\right\}$ & $\left\{\mathrm{k}_{\mathrm{c}}, \mathrm{t}, 4_{\mathrm{c}}\right\}$ \\
\hline LL & $\left\{\mathrm{m}^{+}, \mathrm{n}\right\}$ & $\left\{\mathrm{m}^{+}\right.$, & & $\left\{\mathrm{k}_{c}^{+}\right\}$ & $\left\{k_{c}^{-}\right.$ & & $\left\{\mathrm{k}_{\mathrm{c}}^{+}, \mathrm{d}_{\mathrm{c}}^{+}\right\}$ & $\left\{\mathrm{k}_{\mathrm{c}}^{+}, \mathrm{d}_{\mathrm{c}}\right\}$ & $\left\{k_{c}^{+}\right\}$ & $\left\{\mathrm{k}_{\mathrm{c}}^{+}\right\}$ \\
\hline$X^{\downarrow}$ & $\left\{t^{\downarrow}\right\}$ & $\left\{t^{\downarrow}, 4\right.$ & & $\varnothing$ & $\{4$ & & $\varnothing$ & $\left\{4^{\downarrow}\right\}$ & $\left\{t^{\downarrow}\right\}$ & $\left\{t^{\downarrow}, 4^{\downarrow}\right\}$ \\
\hline
\end{tabular}

Fig. 13. Rule sets for the S4-tesseract logics not given in Figure 7

\begin{tabular}{l|c|l}
$\mathrm{X}$ & $\mathrm{X}^{\mathrm{LL}}$ & for each $\vee$-equivalence class $\rho$ in $V^{\square} \uplus V^{\diamond}$ \\
\hline $\mathrm{M}, \mathrm{MT}, \mathrm{M} 4, \mathrm{MT} 4$ & $\left\{\mathrm{~m}^{+}\right\}$ & $\rho=\{u, v\}$ with $u \in V^{\square}$ and $v \in V^{\diamond}$ \\
$\mathrm{MP}, \mathrm{MP4}$ & $\left\{\mathrm{m}^{+}, \mathrm{p}\right\}$ & $\rho=\{u, v\}$ or $\rho=\{v\}$ with $u \in V^{\square}$ and $v \in V^{\diamond}$ \\
$\mathrm{MD}, \mathrm{MD} 4$ & $\left\{\mathrm{~m}^{+}, \mathrm{d}_{\mathrm{m}}^{+}\right\}$ & $\rho=\{u, v\}$ with $v \in V^{\diamond}$ \\
$\mathrm{N}, \mathrm{NT}, \mathrm{N} 4, \mathrm{NT} 4$ & $\left\{\mathrm{~m}^{+}, \mathrm{n}\right\}$ & $\rho=\{u, v\}$ or $\rho=\{u\}$ with $u \in V^{\square}$ \\
$\mathrm{NP}, \mathrm{NP4}$ & $\left\{\mathrm{m}^{+}, \mathrm{n}, \mathrm{p}\right\}$ & $\rho=\{u, v\}$ or $\rho=\{w\}$ with $u \in V^{\square}$ and $v \in V^{\diamond}$ \\
$\mathrm{ND}, \mathrm{ND4}$ & $\left\{\mathrm{m}^{+}, \mathrm{n}, \mathrm{d}_{\mathrm{m}}^{+}\right\}$ & $\rho=\{u, v\}$ or $\rho=\{w\}$ with $v \in V^{\diamond}$ and $w \in V^{\square}$ \\
$\mathrm{C}, \mathrm{CT}, \mathrm{C4}, \mathrm{CT} 4$ & $\left\{\mathrm{k}_{\mathrm{c}}^{+}\right\}$ & $\rho=\left\{u, v, w_{1}, \ldots, w_{n}\right\}$ with $u \in V^{\square}$ and $v, w_{1}, \ldots, w_{n} \in V^{\diamond}$ \\
$\mathrm{CD}, \mathrm{CD} 4$ & $\left\{\mathrm{k}_{\mathrm{c}}^{+}, \mathrm{d}_{\mathrm{c}}^{+}\right\}$ & $\rho=\left\{u, v, w_{1}, \ldots, w_{n}\right\}$ with $v, w_{1}, \ldots, w_{n} \in V^{\diamond}$ \\
$\mathrm{K}, \mathrm{KT}, \mathrm{K} 4, \mathrm{KT} 4$ & $\left\{\mathrm{k}^{+}\right\}$ & $\rho=\left\{u, v_{1}, \ldots, v_{n}\right\}$ with $u \in V^{\square}$ and $v_{1}, \ldots, v_{n} \in V^{\diamond}$ \\
$\mathrm{KD}, \mathrm{KD} 4$ & $\left\{\mathrm{k}^{+}, \mathrm{d}^{+}\right\}$ & $\rho=\left\{u, v_{1}, \ldots, v_{n}\right\}$ with $v_{1}, \ldots, v_{n} \in V^{\diamond}$
\end{tabular}

Fig. 14. The fourth condition an RGB-cograph has to satisfy in order to be $\mathrm{X}^{\mathrm{LL}}$-correct.

Definition 8.2 Let $X$ be a logic in the S4-tesseract. An RGB-cograph is $\mathrm{X}^{\mathrm{LL}}$ correct, if it obeys Conditions 13 of Definition 4.3. together with the corresponding version of Condition 4 shown in the table in Figure 14.

Intuitively, the corresponding conditions in the table in Figure 14 verify if the number of $\square$ - and $\diamond$-vertices in an $\checkmark$-equivalence class is compatible with the number of $\square$ - and $\diamond$-occurrences introduced in a sequent by a single application of a sequent rule of $\mathrm{X}^{\mathrm{LL}}$.

With this we can show Theorem 4.4 for all logics in the S4-tesseract shown in Figure 1. Therefore, X-combinatorial proofs, as defined in Definition 7.7 form a sound and complete proofs system for the modal logic $X$, as stated in Theorems 6.3, 6.4 and 6.5. for all logics $X$ in the S4-tesseract shown in Figure 1 . 


\section{Conclusion and Future Work}

In this paper we presented cominatorial proofs for all logics in the S4-tesseract. Since checking correctness of a combinatorial proof is polynomial in its size, they form a proof system in the sense of Cook and Reckhow 9. Due to their combinatorial nature, they abstract away from the syntactic bureaucracy of more standard formalisms like sequent calculus or analytic tableaux. This leads naturally to the following notion of proof identity:

Two proofs are the same iff they have the same combinatorial proof.

We conjecture that this notion of proof identity is in close correspondence to the notion of proof identity that is induced by sequent rule permutations. However, investigating the relation between the two would go beyond the scope of this paper, and we consider this to be future work. Furthermore, in order to support (13) it is necessary, not only to show how sequent proofs are related to combinatorial proofs, but also how analytic tableaux or resolution proofs or other syntactic formalisms are related to combinatorial proofs [1].

Further topics for future work include the extensions to all logics in the classical modal S5-cube, and also to intuitionistic modal logics. Another question is how our work relates to the recent development of combinatorial proofs for first-order logic [17.

Finally, from the proof theoretical perspective, the most interesting question for future research is the study of normalization of combinatorial proofs, as it has been done for propositional logic in [16/29].

\section{References}

1. Acclavio, M., Straßburger, L.: From syntactic proofs to combinatorial proofs. In: International Joint Conference on Automated Reasoning. pp. 481-497. Springer (2018)

2. Acclavio, M., Straßburger, L.: On combinatorial proofs for logics of relevance and entailment. In: International Workshop on Logic, Language, Information, and Computation. pp. 1-16. Springer (2019)

3. Avron, A.: The method of hypersequents in the proof theory of propositional nonclassical logics. In: Logic: from foundations to applications, European Logic Colloquium. pp. 1-32. Oxford University Press (1994)

4. Benjamin, R., Straßburger, L.: Towards a combinatorial proof theory. In: Tableaux 2019. Springer (2019)

5. Brünnler, K.: Deep sequent systems for modal logic. Archive for Mathematical Logic 48(6), 551-577 (2009)

6. Brünnler, K., Tiu, A.F.: A local system for classical logic. In: Nieuwenhuis, R., Voronkov, A. (eds.) LPAR 2001. LNAI, vol. 2250, pp. 347-361. Springer (2001)

7. Chaudhuri, K., Marin, S., Straßburger, L.: Focused and synthetic nested sequents. In: FoSSaCS'16. pp. 390-407. Springer (2016)

8. Chaudhuri, K., Marin, S., Straßburger, L.: Modular Focused Proof Systems for Intuitionistic Modal Logics. In: Kesner, D., Pientka, B. (eds.) FSCD'16. LIPIcs, vol. 52, pp. 16:1-16:18. Schloss Dagstuhl-Leibniz-Zentrum fuer Informatik (2016) 
9. Cook, S.A., Reckhow, R.A.: The relative efficiency of propositional proof systems. J. of Symb. Logic 44(1), 36-50 (1979)

10. Goré, R., Postniece, L., Tiu, A.: On the correspondence between display postulates and deep inference in nested sequent calculi for tense logics. Logical Methods in Computer Science 7(2) (2011)

11. Goré, R., Ramanayake, R., et al.: Labelled tree sequents, tree hypersequents and nested (deep) sequents. Advances in modal logic 9, 279-299 (2012)

12. Guglielmi, A.: A system of interaction and structure. ACM Transactions on Computational Logic 8(1), 1-64 (2007)

13. Guglielmi, A., Straßburger, L.: Non-commutativity and MELL in the calculus of structures. In: CSL 2001. LNCS, vol. 2142, pp. 54-68. Springer (2001)

14. Hein, R., Stewart, C.: Purity through unravelling. Structures and Deduction pp. 126-143 (2005)

15. Hughes, D.: Proofs Without Syntax. Annals of Math. 164(3), 1065-1076 (2006)

16. Hughes, D.: Towards Hilbert's $24^{\text {th }}$ problem: Combinatorial proof invariants: (preliminary version). Electr. Notes Theor. Comput. Sci. 165, 37-63 (2006)

17. Hughes, D.J.D.: First-order proofs without syntax (Jun 2019)

18. Lellmann, B.: Hypersequent rules with restricted contexts for propositional modal logics. Theoretical Computer Science 656, 76-105 (2016)

19. Lellmann, B., Pimentel, E.: Modularisation of sequent calculi for normal and nonnormal modalities. ACM Transactions on Computational Logic (TOCL) 20(2), 7 (2019)

20. Marin, S., Straßburger, L.: Label-free Modular Systems for Classical and Intuitionistic Modal Logics. In: Advances in Modal Logic 10 (2014)

21. Möhring, R.H.: Computationally tractable classes of ordered sets. In: Rival, I. (ed.) Algorithms and Order, pp. 105-194. Kluwer (1989)

22. Negri, S.: Proof analysis in modal logic. Journal of Philosophical Logic 34(5-6), 507 (2005)

23. Retoré, C.: Pomset logic: a non-commutative extension of classical linear logic. In: TLCA 1997. pp. 300-318. Springer (1997)

24. Retoré, C.: Handsome proof-nets: perfect matchings and cographs. Theor. Comput. Sci. 294(3), 473-488 (2003)

25. Simpson, A.K.: The proof theory and semantics of intuitionistic modal logic. Ph.D. thesis, University of Edinburgh. College of Science and Engineering (1994)

26. Stewart, C., Stouppa, P.: A systematic proof theory for several modal logics. Advances in modal logic 5, 309-333 (2004)

27. Straßburger, L.: A characterization of medial as rewriting rule. In: RTA'07. vol. 4533, pp. 344-358. Springer (2007)

28. Straßburger, L.: Cut elimination in nested sequents for intuitionistic modal logics. In: Pfenning, F. (ed.) FoSSaCS'13. LNCS, vol. 7794, pp. 209-224. Springer (2013)

29. Straßburger, L.: Combinatorial flows and their normalisation. In: Miller, D. (ed.) FSCD'17. LIPIcs, vol. 84, pp. 31:1-31:17. Schloss Dagstuhl (2017)

30. Straßburger, L.: The problem of proof identity, and why computer scientists should care about Hilbert's 24th problem. Philosophical Transactions of the Royal Society A 377(2140), 20180038 (2019)

31. Thiele, R.: Hilbert's twenty-fourth problem. American Math. Monthly 110, 1-24 (2003)

32. Wansing, H.: Sequent calculi for normal modal propositional logics. Journal of Logic and Computation 4(2), 125-142 (1994)

33. Wansing, H.: Sequent systems for modal logics. In: Handbook of philosophical logic, pp. 61-145. Springer (2002) 